\title{
Equilibrium and kinetics at the coil-to-globule transition of star and comb heteropolymers in infinitely dilute solutions
}

\author{
Edward G. Timoshenko* \\ Theory and Computation Group, Department of Chemistry, University College Dublin, Belfield, Dublin 4, Ireland \\ Yuri A. Kuznetsov ${ }^{\dagger}$ \\ Centre for High Performance Computing Applications, University College Dublin, Belfield, Dublin 4, Ireland
}

(November 15, 2018)

\begin{abstract}
By means of continuous space Monte Carlo simulation we study conformational structures formed by star and comb heteropolymers during kinetics of folding from the coil to the globule, as well as the corresponding equilibrium states on going from the good to the poor solution. Particular examples of combs with hydrophobic backbone and hydrophilic side-groups (and vice versa), as well as stars with flexible and semi-stiff arms are studied. It is interesting to note that star-like conformations naturally appear for a comb polymer with a strongly hydrophobic backbone. We emphasise the crucial difference in the spatial distribution of hydrophobic and hydrophilic monomers within the globular states for the above mentioned two types of combs. In case of stars, the non-equilibrium states during kinetics of the coil-to-globule transition correspond to formation of localised pearls within flexible arms, whereas semi-stiff arms prefer to join with each other remaining essentially extended. These studies present natural extension of our previous works on the equilibrium and kinetic properties of linear and ring heteropolymers based on the Gaussian self-consistent method and lattice Monte Carlo technique. However, in studying polymers with more nontrivial topology continuous space simulation has some essential advantages.
\end{abstract}

Keywords: heteropolymer, conformation, kinetics, star, comb

\section{INTRODUCTION}

From the polymer design perspective three properties of a polymer are of major importance for the resulting conformational properties and consequent industrial applications. These are the degree of stiffness of the chain, the topology of the polymer, and its composition in terms of monomers involved. In trying to model, for instance, the structure of a natural protein one has to construct the main chain of a given stiffness and to attach various side chains, each of which would have required local stiffness, degree of hydrophobicity and other more specific properties associated with the nature of the corresponding amino acid residue. The challenging nature of the kinetics of protein folding would manifest itself in the shear complexity and diversity of the kinetics of folding of our hypothetical model polymer, which should be rather sensitive on the details of these three types of interactions involved. In the current paper we shall discuss the results from Monte Carlo simulations in continuous space for the kinetics of folding of several sufficiently simple and yet already complex types of polymers based on a rather generic coarse-grained model introduced here. These preliminary studies of the model produce a number of novel transient, and in some cases metastable, kinetic intermediates and rather complex kinetic pathways of folding.

Conformational transitions of homopolymers with varying degree of flexibility have been of considerable interest for analytical studies [1,2] and computer simulations [3, 4 in in recent years. It is well known that folding of a sufficiently stiff chain results in formation of a torus [5] under certain conditions. Moreover, the transitions coil-to-torus and torus-to-globule are discontinuous, resulting in a rather complex kinetic picture of expansion or collapse, essentially dependent on the quench depth [6]. The phase diagram of a stiff homopolymer obtained from the Gaussian variational method [6] is found to contain phases of the coil, a spherical (or slightly aspherical) globule in the region of either a low stiffness or a strong monomer attraction, and a number of toroidal phases characterised by distinct winding number in between. The collapse transition changes its behaviour from continuous to discontinuous starting from some value of the stiffness. This distinction is quite dramatic for the kinetics of collapse. While the kinetics of the coil-to-globule transition for a flexible homopolymer proceeds via formation of a necklace of locally collapsed sub-globules or 'pearls' [7] rather than via a sausage mechanism [8], the pearls are not seen in the semi-flexible case where hairpins with

*Author to whom correspondence should be addressed. Phone: +353-1-7162821, Fax: +353-1-7162127. Web page: http://darkstar.ucd.if; E-mail: Edward.Timoshenko@ucd.ie

${ }^{\dagger}$ E-mail: Yuri.Kuznetsov@ucd.ie 
abrupt U-turns and indeed sausages, often also corresponding to metastable states, would be typical transient kinetic intermediates [6].

There is also a great deal of interest in the properties of amphiphilic heteropolymers. Even in the flexible case these exhibit a variety of complex conformational transitions upon a temperature or $\mathrm{pH}$ variation 9 [1]. Apart from the stiffness and the sequence of monomers, one can also play with various polymer topologies 12$]$. Indeed, random branching of polymers is quite common during synthesis. By a carefully controlled polymerisation procedure one can obtain regular stars, which presented a nice object for theoretical study due to their symmetry and simplicity lately [1],13]. Star polymers are believed to find a number of future applications for coating, as additives and possibly in drug delivery systems related to their low viscosity and other interesting properties.

Speaking of computer simulations, star polymers, for instance, (see e.g. Refs. [14,15]) have been studied by a number of techniques, ranging from Monte Carlo, both on and off lattice, to Brownian and Molecular Dynamics [15]. While dynamic Monte Carlo is one of perhaps most efficient approaches in terms of accessible time-scales, on a a lattice it has a number of obvious limitations. Indeed, the core monomer on a lattice has a great difficulty to move at all due to its high coordination number (equal to the number of arms). The resulting rejection of all attempted moves for some kind of motions is called a quasi-nonergodicity, a problem which is hard to deal with even for linear copolymers. If such a situation occurs, it practically means that the results of the simulation may no longer be trusted. A number of complicated non-local moves involving the core monomer have been suggested to alleviate this problem [16]. However, such moves are not permitted in case of Dynamic Monte Carlo needed for study of, for instance, metastable states, moreover they may present problems due to subtle topological effects for truly non-phantom chains 17].

Referring to our recent paper [13] for examples of applications to diblock copolymer star, we devote the present work to investigating the kinetics of the coil-to-globule transition for flexible and semi-flexible homopolymer stars and diblock copolymer combs with the hydrophobic backbone (or H-backbone comb briefly) with hydrophilic (polar) side-arms and vice versa (or P-backbone comb).

\section{RESULTS}

The mathematical model for a generic coarse-grained polymer is based upon the following Hamiltonian (energy functional):

$$
\begin{aligned}
H= & \frac{k_{B} T}{2 \ell^{2}} \sum_{i \sim j} \kappa_{i j}\left(\mathbf{X}_{i}-\mathbf{X}_{j}\right)^{2}+\frac{k_{B} T}{2 \ell^{2}} \sum_{i \approx j \approx k} \lambda_{i j k}\left(\mathbf{X}_{i}+\mathbf{X}_{k}-2 \mathbf{X}_{j}\right)^{2} \\
& +\frac{1}{2} \sum_{i j, i \neq j} V_{i j}\left(\left|\mathbf{X}_{i}-\mathbf{X}_{j}\right|\right) .
\end{aligned}
$$

Here the first term presents the connectivity structure of the polymer with harmonic springs of a given strength $\kappa_{i j}$ introduced between any two connected monomers (which is denoted as $i \sim j$ ). The second term presents the bending energy penalty given by the square of the local curvature with a characteristic stiffness $\lambda_{i j k}$ between any three consecutively connected monomers (which is denoted as $i \approx j \approx k$ ) in the form of the Kratky-Harris-Hearst term [18, 19]. Finally, the third term presents pair-wise non-bonded interactions between monomers such as van der Waals forces and so on. In a simple model we can adopt the Lennard-Jones form for the shape of the potential,

$$
V_{i j}(r)=\left\{\begin{array}{ll}
+\infty, & r<d \\
V_{i j}^{0}\left(\left(\frac{d}{r}\right)^{12}-\left(\frac{d}{r}\right)^{6}\right), & r>d
\end{array},\right.
$$

where there is also a hard core part with the monomer diameter $d$ (below this is taken to be equal to the statistical length $\ell$, i.e $d=\ell$ ). Finally, we shall restrict ourselves to a binary heteropolymer with hydrophilic and hydrophobic units, so that the potential coefficients can be further parametrised as [20],

$$
V_{i j}^{0}=\frac{V_{i}+V_{j}}{2}, \quad V_{i}=V_{0}\left(1-\sigma_{i}\right), \quad \sigma_{i}= \pm 1 .
$$

For details of the adopted Monte Carlo technique we refer to our paper Ref. [6].

Our first subject is the collapse kinetics of a flexible $(\lambda=0)$ homopolymer star after a quench from the purely repulsive good solvent condition $\left(V_{0}=0\right)$ to the poor solvent $\left(V_{0}=5\right)$. Here and below $k_{B} T$ units are used for the energy and $\ell$ units for the distance. From Fig. 11a one can see that the process involves formation of locally collapsed clusters along the chain similarly to the case of an open or ring chain. However, due to the connectivity structure 
of the star, the largest cluster is formed around the core monomer and the arms ends seem to belong to fairly large clusters. Also, it is interesting to note that some of the clusters involve monomers from more than one arm (see e.g. on the bottom right of the figure). Further, the ends of arms clusters are being pulled towards the growing core cluster, so that the total number of clusters and free arms rapidly decrease (Fig. 1 b). Finally, an almost spherical globule will form and continue to optimise its shape and internal structure until reaching the final equilibrium exponentially slowly [7].

In Figs. 2, similarly, we examine the kinetic process for a semi-flexible homopolymer star with $\lambda=5$. Clearly, as in the case of a semi-flexible rings in Ref. [6] formation of the localised clusters is no longer possible due to a large energetic penalty. Instead, as in Fig. $2 \mathrm{a}$ some of the arms come together forming a kind of fibrils, some of which still have free outstretched arms. This tree-like structure further experiences the process of fibrils growth with their width increasing and the number and lengths decreasing (Figs. $2 \mathrm{~b}$ and $2 \mathrm{cc}$ ). The overall compaction process is much slower compared to the flexible case and finally a rather long-lived sausage-like object would remain. It is interesting to point out that quite often these kinetically arrested states would have one or a few pieces of single arms sticking out of the the main irregularly shaped body as in Figs. 2lc and 2d. These curious looking conformations are rather typical for a semi-flexible chain and should in principle be seen by Electron Microscopy in experiments involving long semi-stiff star polymers. This effect is clearly related to the topology of a star since similar conformations are not present for a ring polymer [6].

Now let us turn our attention to flexible comb copolymers. We analyse the simple case in which the backbone is constructed of one type of monomers, while the side-arms of another, one of which is hydrophobic (H) and another hydrophilic (P for polar), so that the total numbers of monomers of both kinds are equal. In particular we shall analyse the situation in which in the final state $V_{P P}^{0}=0$ (pure repulsion), $V_{H H}^{0}=5$ and $V_{H P}^{0}=2.5$, corresponding to a near ideal regime for the H-P monomer interaction. In Figs. 3 we present the characteristic equilibrium conformations for the cases of the H-backbone (Fig. 3a) and the P-backbone (Fig. 3b). The former case pretty much reminds the situation for a OUTER-P star [13], so that in Fig. 3a we see the collapsed hydrophobic backbone in the centre with three outstretched hydrophilic side-groups (the forth one is pointing in the perpendicular to the plane direction and thus is not visible). This object can be viewed as a micellar star. The reversed case of the P-backbone again produces a micellar object with a single collapsed core of hydrophobic side-groups and extended hydrophilic backbone chain looping around the core. This is a much more compact object compared to the H-backbone case and it is in many ways similar to the collapsed OUTER-H star in Ref. [13]. Also, the H-core in Fig. 3 $3 \mathrm{~b}$ is clearly much less exposed to unfavourable contacts with the solvent molecules than in Fig. 3 a.

Next, let us also discuss briefly the transient kinetic states after a quench from the good solvent condition (all

$\left.V_{i j}^{0}=0\right)$ to the above discussed final equilibrium values in both cases. In Fig. Ga one can see the formation of locally collapsed clusters along the hydrophobic back backbone, which further grow and merge with each other until producing a single globular core with the hydrophilic side-arms remaining extended and outstretched throughout. This mechanism is akin to the case of an open homopolymer collapse except that perhaps the mobility of the growing clusters is somewhat reduced by the presence of the passive side-arms. Finally, in Fig. đb we present a typical nonequilibrium conformation for the P-backbone comb. Here, the folding process starts very quickly by a full collapse of all individual hydrophobic side-arms, which remain farther apart from each other along the hydrophilic backbone. This arrangement results in a rather long-lived metastable state, which has a rather distinct structure from the final equilibrium state depicted in Fig. 3 $3 \mathrm{~b}$. An example of a similar metastable state in kinetics was also seen for an OUTER-H star in Ref. [13], where we have also seen that a much deeper quench would render such a state unstable turning the nucleation regime into that of spinodal decomposition.

\section{CONCLUSIONS}

In this work we have presented some preliminary results on the studies of the equilibrium and kinetics of conformational changes in flexible and semi-flexible homo- and block copolymer stars and combs. We have seen a number of unusual conformational states and distinct folding pathways strongly dependent on the macromolecular architecture.

Study of the homopolymer collapse kinetics in stars of varying degree of flexibility has elucidated that the persistent length of the chain does play a major role in determining whether the necklace of clusters or the fibril growth mechanism of folding is realised. This was also true for simpler topological objects such as an open chain or a ring [7.6]. However, there are some topology induced and previously unseen features such as for instance a 'monkey and its tail' conformation in Fig. 22d. Further increase of the stiffness may lead to some novel equilibrium phases and a complex resulting phase diagram in analogy with multiple toroidal states having distinct winding numbers for a ring homopolymer [6].

Also, it is interesting that a number of similarities with the previously studied case of diblock star copolymers 13 , 
has been seen here for comb polymers. It is quite likely that many of these features would be also retained for more complex regularly branched dendrimers [21], which are currently being scrutinised.

It would be important to investigate attendant kinetic laws and estimate characteristic time scales involved as we have done for the homopolymer kinetics [7,6, 20]. In doing so, it would be tempting to use several alternative simulation techniques such as a coarse-grained Newton and Langevin Dynamics and an analytical technique based on the Gaussian self-consistent treatment [20] for being able to obtain the most complete picture in this challenging and important problem.

Finally, we believe that modelling chains with distinct types of side-groups is necessary for more realistic description of the kinetics of protein folding. Undoubtedly, a simple heteropolymer model of proteins does miss many of the important interactions and details present in real polypeptides, and a proper account for side-groups and charges of the residues is equally important as the hydrophobic force in driving the folding.

\section{ACKNOWLEDGMENTS}

The authors acknowledge interesting discussions with Professor F. Ganazzoli, Professor H. Orland, Professor T. Garel and Ronan Connolly. This work was supported by grant FR/2000/019 from Enterprise Ireland and UCD President's research award. We also acknowledge the support of the Centre for High Performance Computing Applications, UCD.

[1] F. Ganazzoli, R. La Ferla, G. Allegra, Macromolecules 28 (1995) 5285.

[2] V. V. Vasilevskaya, A. R. Khokhlov, S. Kidoaki, K. Yoshikawa, Biopolymers 41 (1997) 51.

[3] J. P. K. Doye, R. P. Sear, D. Frenkel, J. Chem. Phys. 108 (1998) 2134.

[4] V.A. Ivanov, W. Paul, K. Binder, J. Chem. Phys. 109 (1998) 5659.

[5] A.Yu. Grosberg, Biophysics 24 (1979) 30; A.Yu. Grosberg, A.R. Khokhlov, Adv. Polym. Sci. 41 (1981) 53.

[6] Yu.A. Kuznetsov, E.G. Timoshenko, J. Chem. Phys. 111 (1999) 3744.

[7] E. G. Timoshenko, Yu. A. Kuznetsov, K. A. Dawson, J. Chem. Phys. 102 (1995) 1816.

[8] P. G. de Gennes, J. Phys. Lett. 46 (1985) L639.

[9] K. A. Dill, S. Bromberg, K. Yue, K. M. Fiebig, D. P. Yee, P. D. Thomas, H. S. Chan, Protein Sci. 4 (1995) 561.

[10] D. E. Leckband, O. V. Borisov, A. Halperin, Macromolecules 31 (1998) 2368.

[11] J. Israelachvili, Intermolecular and Surface Forces, Academic, London, 1991.

[12] Y. Rouault, Macromol. Theory Simul. 7 (1998) 359.

[13] F. Ganazzoli, Yu.A. Kuznetsov, E.G. Timoshenko, Macromol. Theory Simul., in press (2000).

[14] G.S. Grest, L. J. Fetters, J. S. Huang, Adv. Chem. Phys. 94 (1996) 67.

[15] J. J. Freire, Adv. Polym. Sci. 143 (1999) 35.

[16] L. A. Molina, J. J. Freire, Macromolecules, 32 (1999) 499.

[17] Yu.A. Kuznetsov, E.G. Timoshenko, Progr. Colloid Polym. Sci. 115 (2000) 146.

[18] O. Kratky, G. Porod, Rec. Trav. Chim. 68 (1949) 1106; H. Yamakawa, Ann. Rev. Phys. Chem. 35 (1984) 23.

[19] R.A. Harris, J.E. Hearst, J. Chem. Phys. 44 (1966) 2595.

[20] E.G. Timoshenko, Yu.A. Kuznetsov, K.A. Dawson, Phys. Rev. E 57 (1998) 6801.

[21] D.A. Tomalia, A.M. Naylor,W.A. Goddard, Angew. Chem. Int. Ed. Engl. 29 (1990) 138. 
FIG. 1. Snapshots of typical non-equilibrium conformations of a flexible star homopolymer during collapse kinetics. This and consequent figures are obtained from the continuous space Monte Carlo simulation. Here the star consists of $f=12$ arms with $N / f=50$ monomers in each arm. Figs. a and b are obtained after $t=20,000$ and $t=100,000$ of Monte Carlo sweeps (MCS) respectively ( $1 \mathrm{MCS}$ is equal to $N+1$ attempted Monte Carlo moves). The kinetics is analysed after the quench of the two-body interaction parameter $V^{0}=0 \rightarrow 5$ (in $k_{B} T$ units).

FIG. 2. Snapshots of typical non-equilibrium conformations of a semi-flexible $(\lambda=5 \ell)$ homopolymer star during collapse kinetics. Figs a $-\mathrm{c}$ are obtained for the same initial condition after $t=35,000, t=350,000$ and $t=10^{6}$ MCS respectively. Fig. $\mathrm{d}$ is obtained from another initial condition after $t=10^{6} \mathrm{MCS}$. Star composition and quench depth here are the same as in Fig. 1.

FIG. 3. Snapshots of typical equilibrium conformations of a flexible comb copolymer. Figs. a and b correspond to the $\mathrm{H}$-backbone and P-backbone combs respectively. Here the number of monomers is $N=288$, the number of side-chains is $f=4$, and the two-body interaction parameters are $\left(V_{P P}^{0}, V_{H P}^{0}, V_{H H}^{0}\right)=(0,2.5,5)$ (in $k_{B} T$ units). Black and light grey circles correspond to hydrophobic and hydrophilic monomers respectively.

FIG. 4. Snapshots of typical non-equilibrium conformations of a flexible comb copolymer during folding kinetics. Figs. a and b correspond to the H-backbone and P-backbone combs respectively. Here the quench depth is $\left(V_{P P}^{0}, V_{H P}^{0}, V_{H H}^{0}\right)=(0,0,0) \rightarrow(0,2.5,5)$ (in $k_{B} T$ units), while the rest of parameters are as in Fig. 3. 


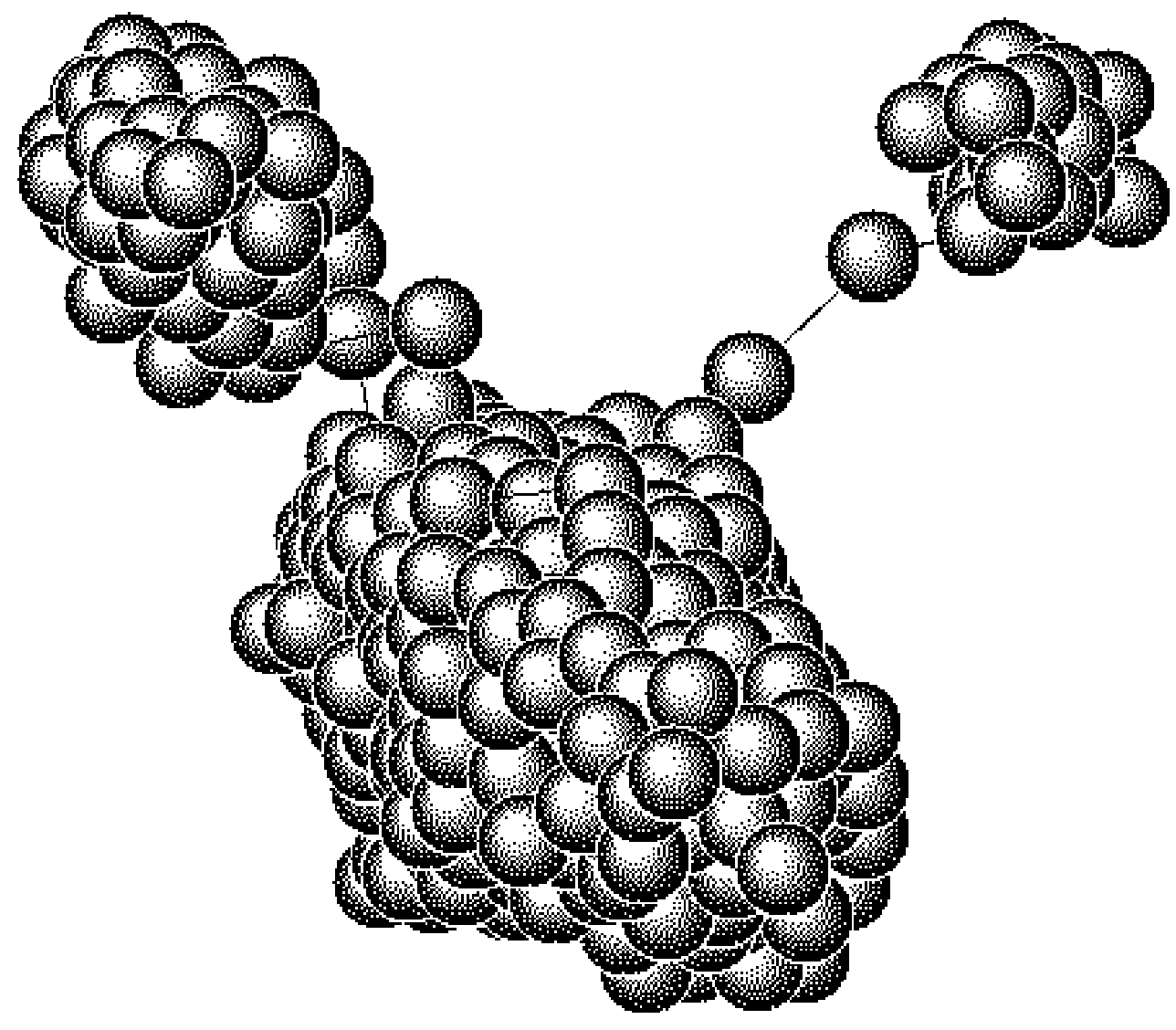

Fig. 1b 


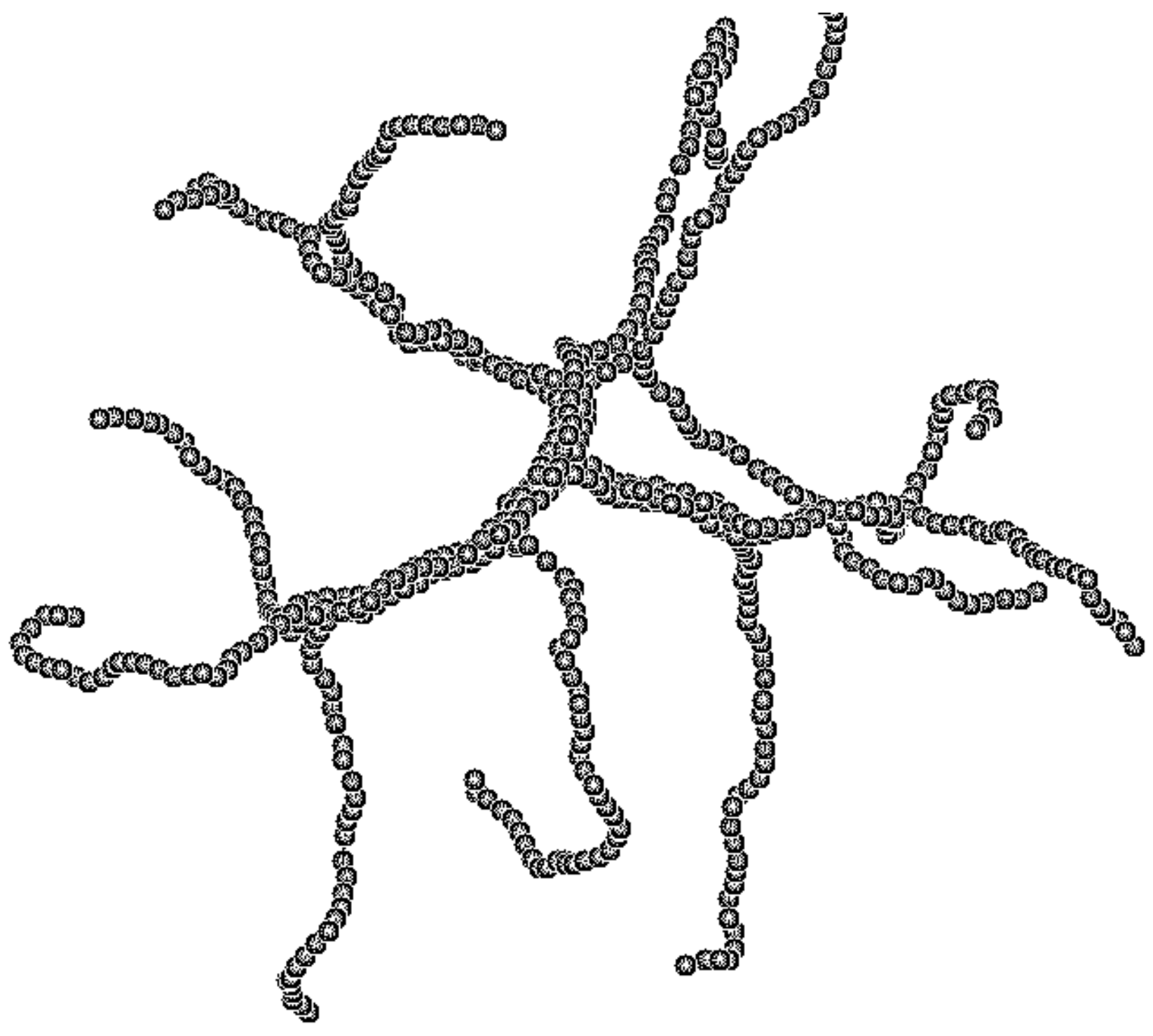

Fig. 2a 


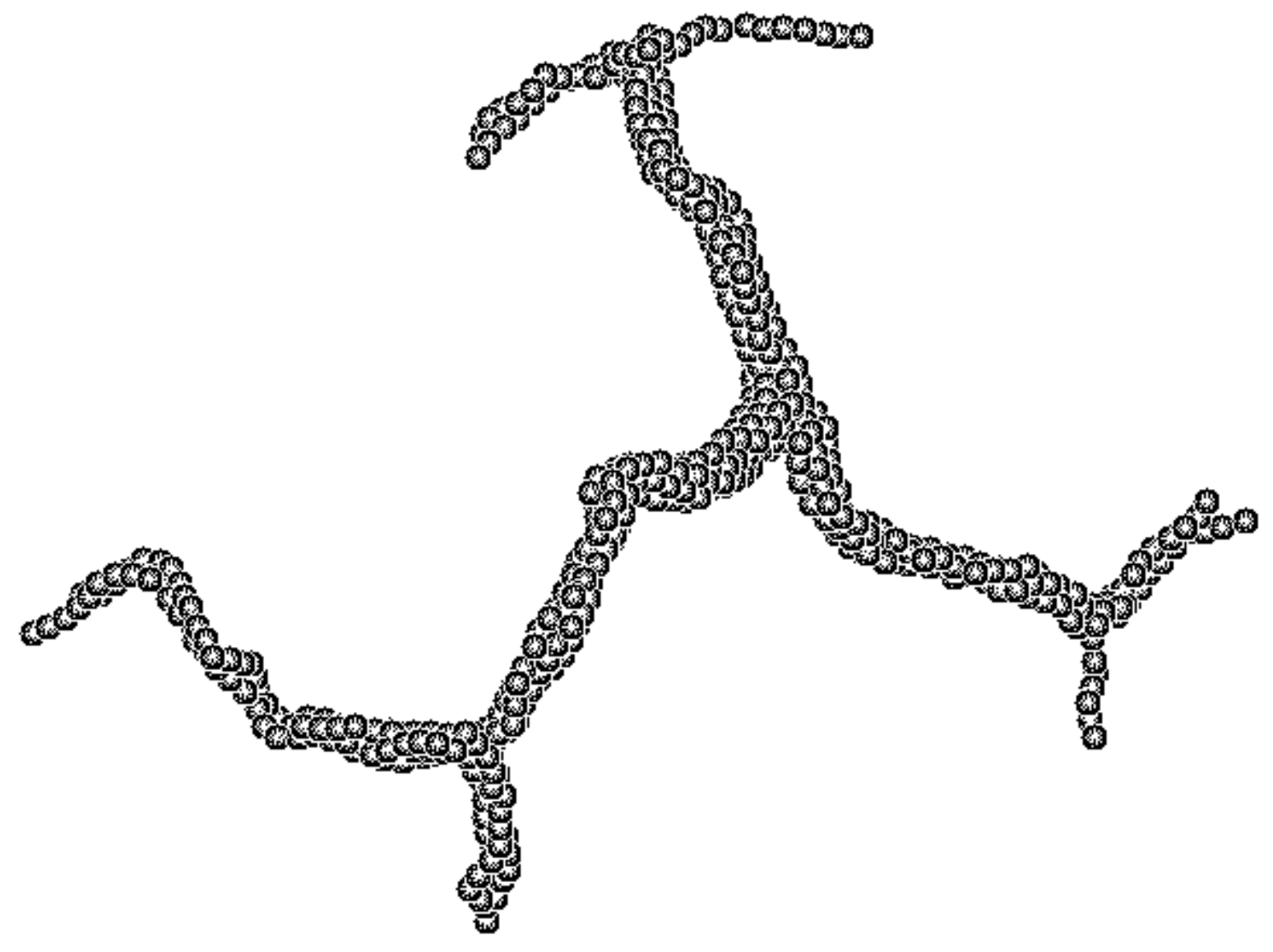

Fig. 2b 


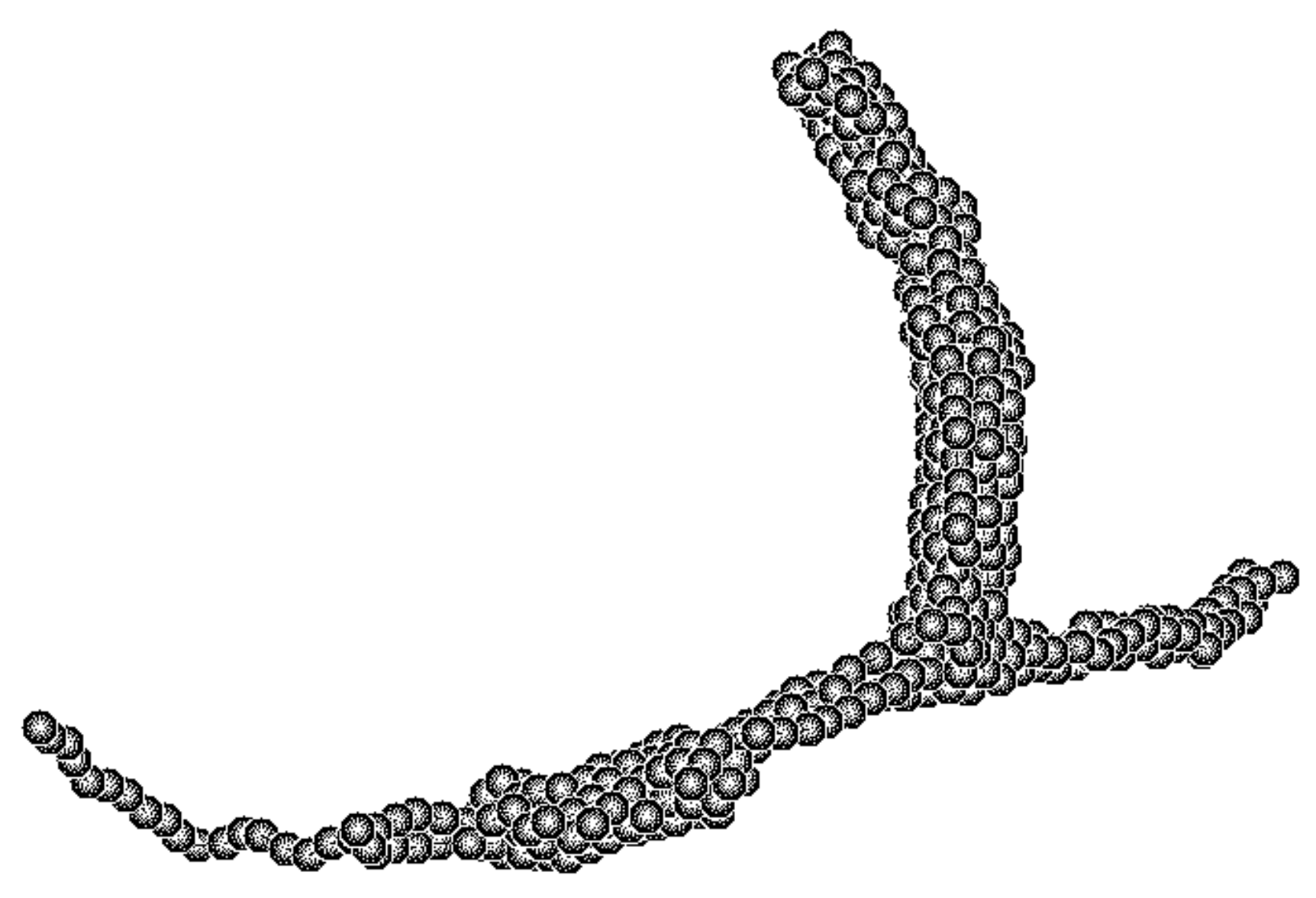

Fig. 2c 


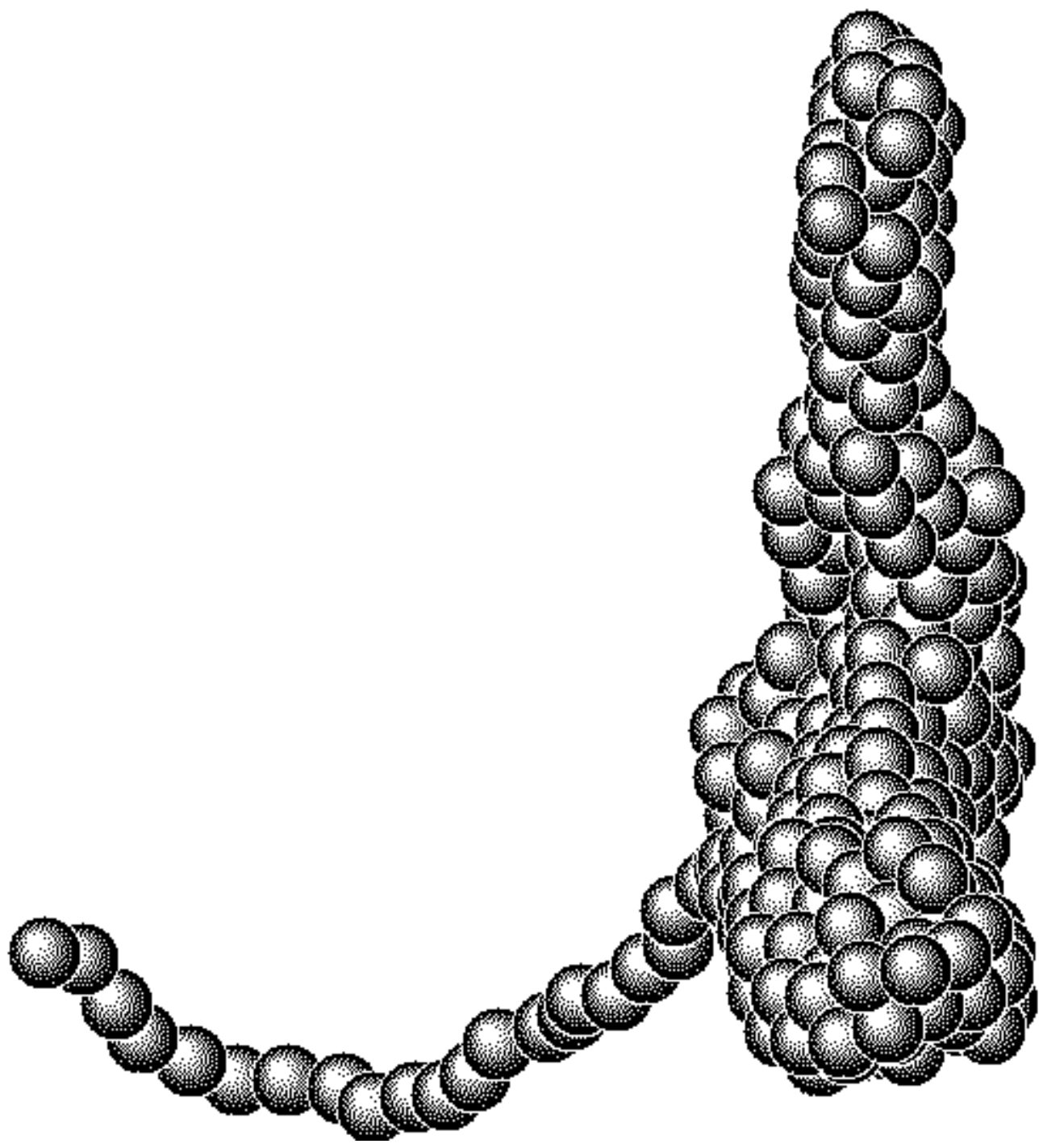

Fig. 2d 


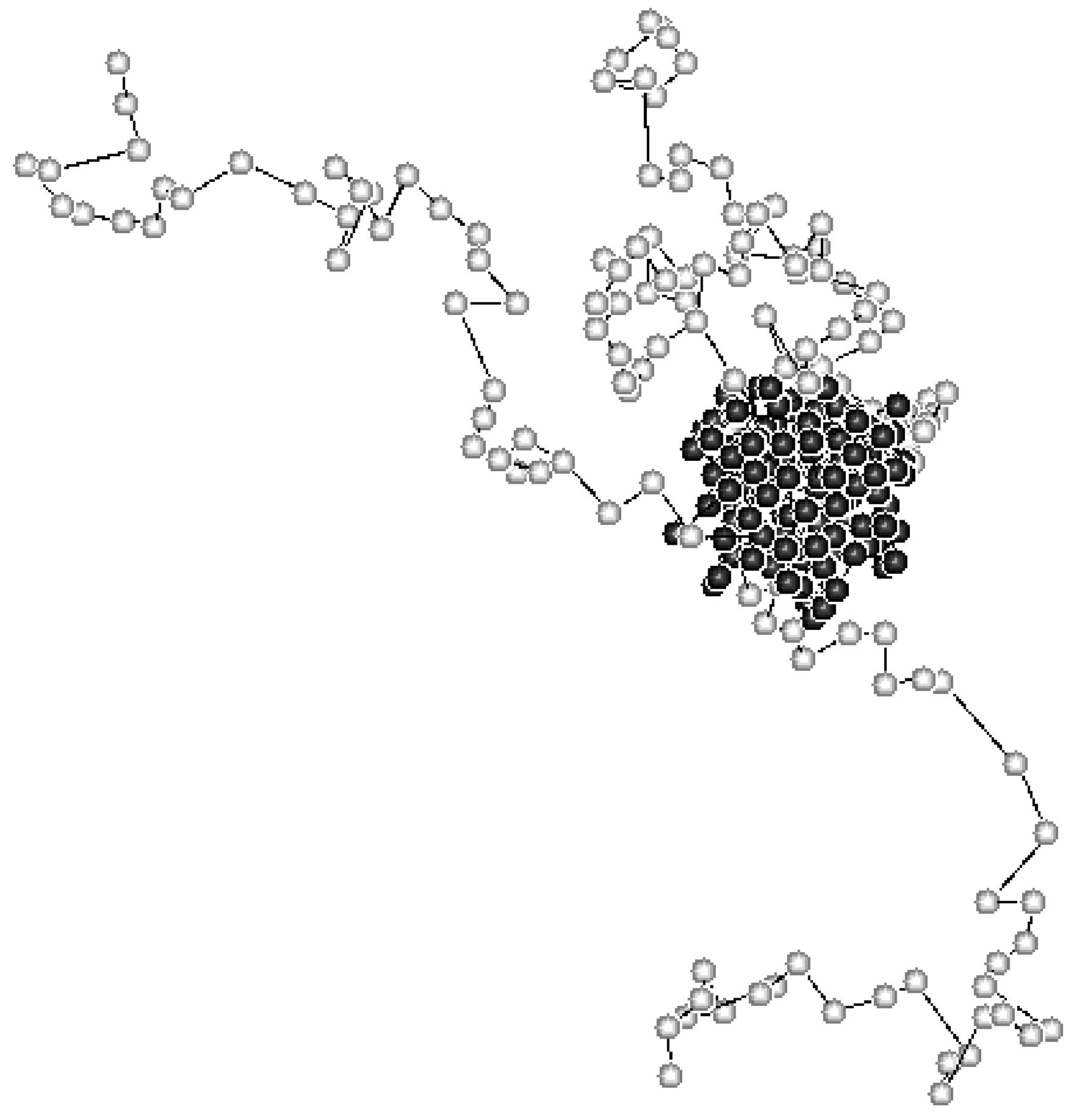

Fig. 3a 


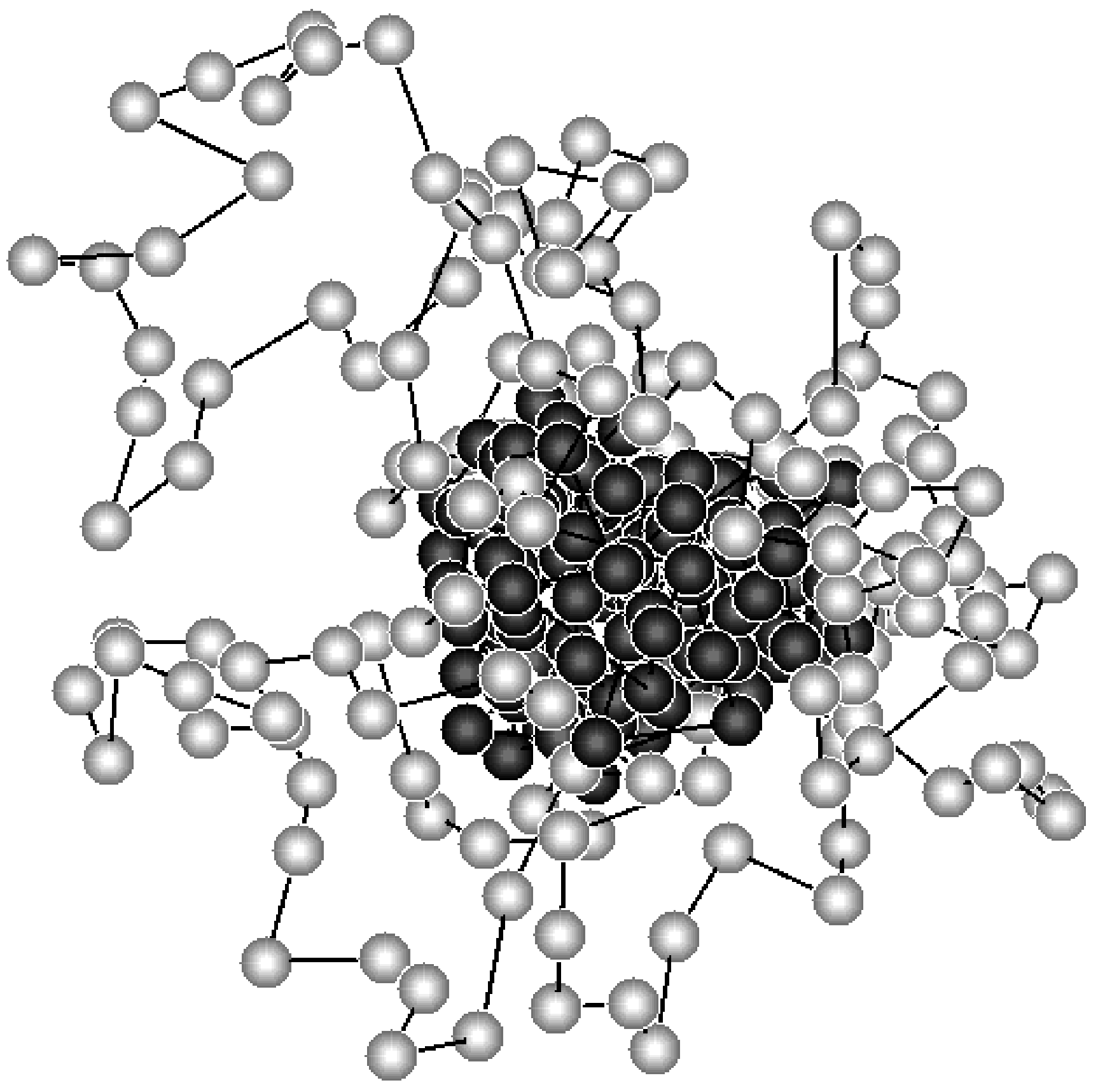

Fig. 3b 


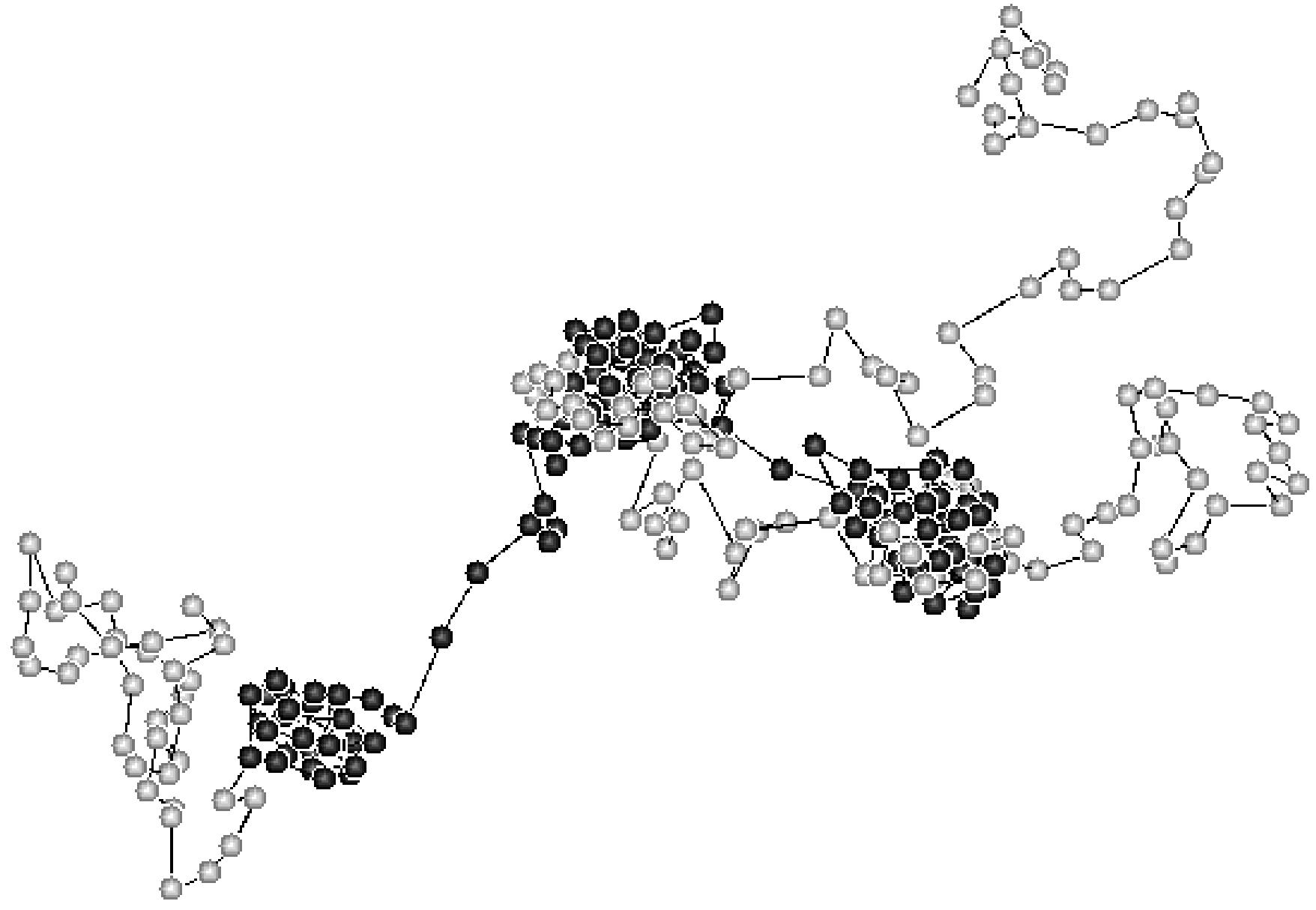

Fig. 4a 


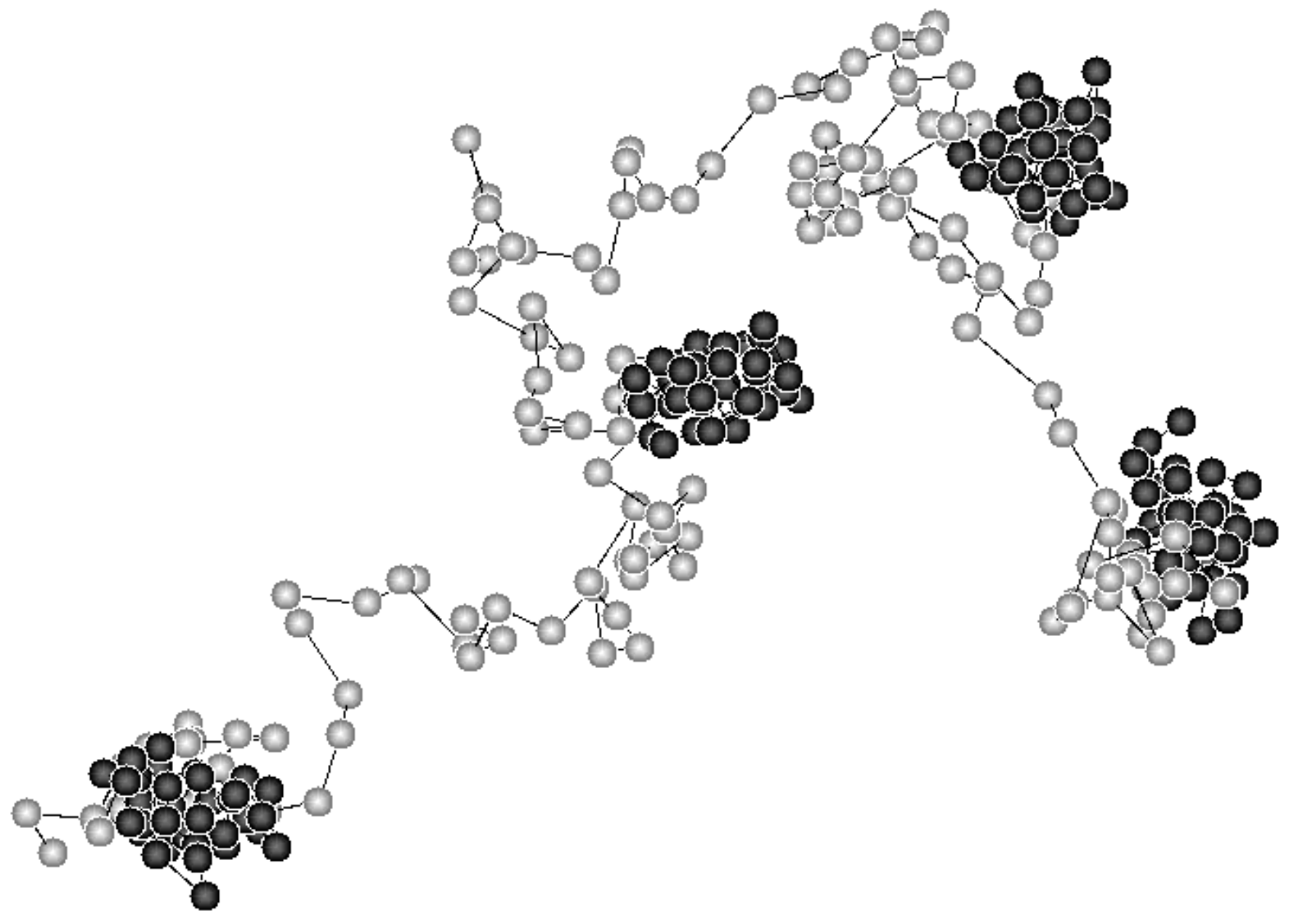

Fig. 4b 